\title{
Camera Embedded Single Lumen Tube as a Rescue Device for Airway Handling during Lung Separation
}

Holm $\mathrm{JH}^{*}$ and Andersen C

Department of Anesthesiology and Intensive Care, Odense University Hospital, Odense, Denmark

${ }^{*}$ Corresponding author: Holm JH, Department of Anesthesiology and Intensive Care, Odense University Hospital, Sdr. Boulevard 29, 5000 Odense C, Denmark, Fax: +45 6590 7231; Tel: +45 6541 4482, E-mail: jimmy.hoejberg.holm@rsyd.dk

Citation: Holm JH, Andersen C (2016) Camera Embedded Single Lumen Tube as a Rescue Device for Airway Handling during Lung Separation. J Case Rep Stud 4(3): 302. doi: 10.15744/2348-9820.4.302

Received Date: February 13, 2016 Accepted Date: June 17, 2016 Published Date: June 20, 2016

\begin{abstract}
Lung isolation in thoracic surgery will usually be achieved either with a double-lumen tube (DLT) or a bronchial blocker (BB). However, even when conducted by anesthesiologists with particular interest and expertise in thoracic anesthesia, the procedure may be troublesome and time consuming.

We report a case of unexpected technical difficulties when isolating the lung in pulmonary surgery for lung cancer, a problem that could lead to cancellation of surgery.

In our case the difficulties were overcome with the use of a camera embedded endotracheal tube (ET) allowing the procedure to proceed.

Keywords: Thoracic anesthesia; Airway handling; VivaSight; Vivasight-SL; Lobectomy; Camera-embedded tube; Endotracheal; Lung isolation; Video tube

List of Abbreviations: DLT: Double Lumen Tube; SLT: Single Lumen Tube; BBs: Bronchial Blockers; ETs: Endotracheal Tubes
\end{abstract}

\section{Case Description}

A 70-year-old woman was scheduled for left posterolateral thoracotomy with lobectomy due to lung cancer. The patient, being a rather small woman, $159 \mathrm{~cm}$ and $50 \mathrm{~kg}$, a small airway was to be expected.

Taking the small stature into account, use of a small conventional 35-Fr right sided DLT was planned for the procedure. As it turned out, this tube could not be passed beyond the vocal cords because too much resistance was felt. We therefore changed to a smaller DLT, and as a DLT size 28-Fr is only available in a left sided version [1], we opted for this. Unfortunately it turned out, that our fiberoptic broncoscope could not be advanced through an ET of this size, and therefore visual verification of correct placement of the tube for lung isolation could not be achieved. As relying on clinical criteria alone for verification of tube placement is not an option in our department, a change to a SLT was opted for, and we decided to use a small diameter single lumen endotracheal tube (SLT, ID $7.0 \mathrm{~mm}$, OD $10.0 \mathrm{~mm}$ ) with embedded camera (VivaSight-SL ${ }^{\mathrm{TM}}$, ET-View Ltd, Misgav, Israel) (Figure 1), and with this secured in the trachea, lung isolation was obtained with the use of an bronchial blocker (VivaSight-EB, 9 Fr) on the left side, resulting in total lung collapse, allowing for the surgery to proceed (Figure 2). The rest of procedure was uneventful with normal one-lung ventilation and a smooth awakening and extubation.

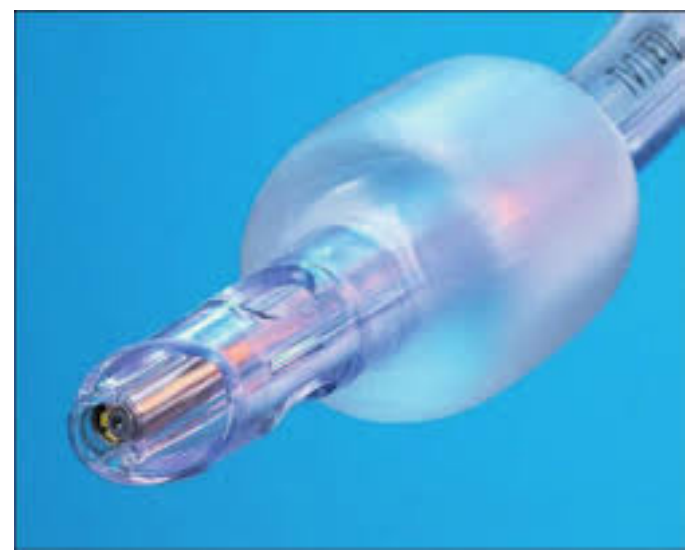

Figure 1: The ET View Camera-Embedded Single Lumen Endotracheal Tube 


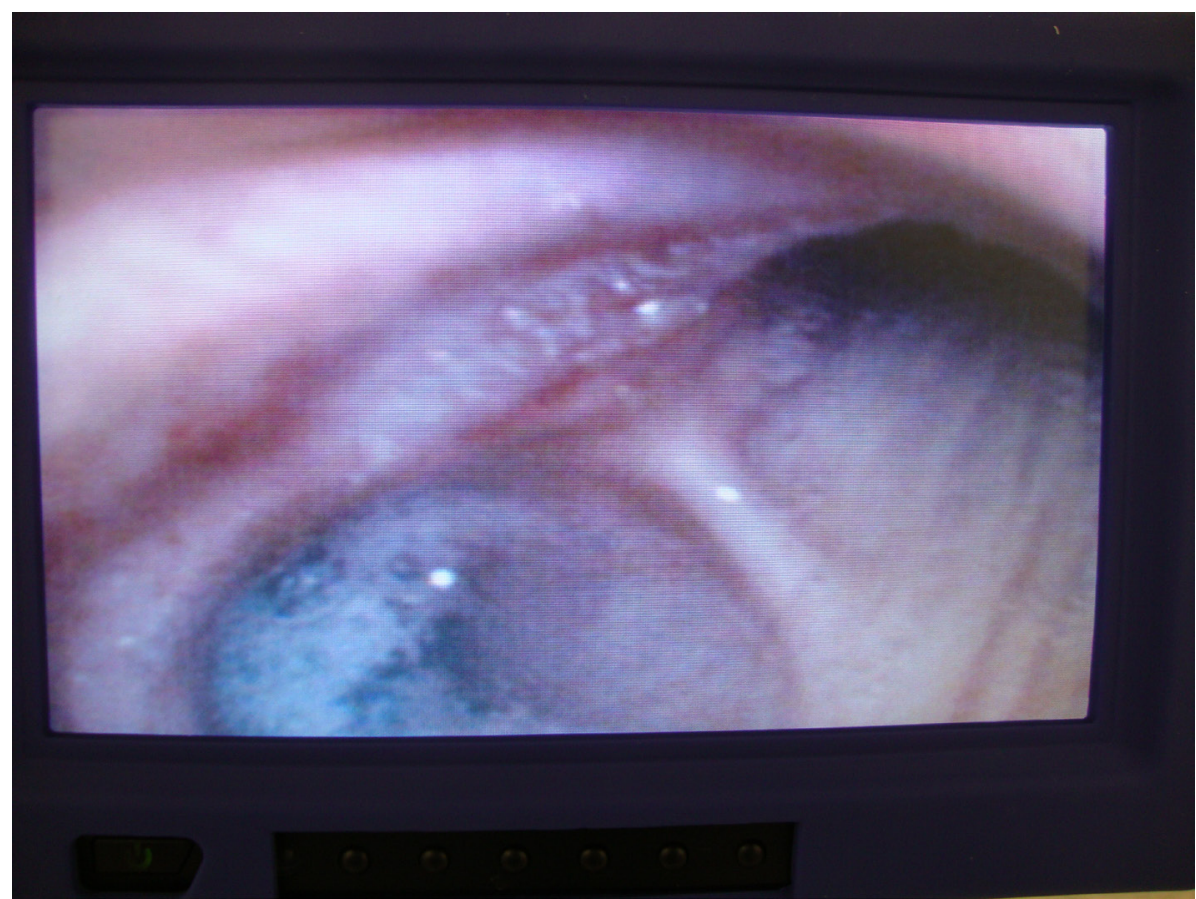

Figure 2: Visual confirmation of correct placement of the bronchial blocker in the left main bronchus

\section{Discussion}

We present a case illustrating the use of camera embedded ET and BB in combination for lung separation, a viable option also for the non-cardiothoracic anesthesiologists.

Normally lung isolation in thoracic anesthesia is handled by anesthesiologists who perform this procedure on a routine basis using either BBs or DLTs. Though a DLT is reported to be the preferred technique for providing lung isolation, the BB is still in routine use [2], both techniques having its advantages and disadvantages.

Most importantly, both types of devices achieve good lung collapse, providing the surgeon with a quiet operative field and protecting the healthy lung from cross-contamination. In a study by Narayanaswamy et al. [3], lung isolation quality of three different BBs was compared with that of DLTs, and in this study no differences were observed by the surgeons.

The DLT offers advantages in patients with significant bleeding or infection, where it offers improved conditions for airway suction. Possible disadvantages are due to the DLT being stiffer and bulkier than a SLT and tracheobronchial injury has been reported with its use [4].

The most significant advantage of the BB over the DLT is that they are placed via a single lumen tube, obviating the need for tube exchange at the end of the procedure should postoperative ventilation be necessary [4]. Other advantages of BBs are the possibility of selective lobar blockade and its use in the presence of a tracheostomy.

A major disadvantage with the BB is the risk of displacement. Displacement will allow re-expansion of the collapsed lung, and more seriously, a herniation of the blockers balloon into the trachea might obstruct ventilation to both lungs, possibly resulting in life-threatening consequences if unrecognized [5].

A disadvantage in the use of both DLTs and BBs is the need for experience in handling fiberoptic equipment. Most BBs require a flexible bronchoscope for placement and as studies have shown, relying on clinical criteria such as observing the expansion of the chest or auscultation alone for placement of DLTs, has an inherent large margin of error of almost 50\% [6].

With the development of new ETs with integrated camera, a whole new range of possibilities has emerged for the anesthesiologist performing one-lung ventilation and possibly more so for the anesthesiologist not experienced in thoracic anesthesia as it might allow for easier lung isolation. When, as in our case, placement of a DLT is not possible or if the anesthesiologist is not sufficiently trained in the use of these, a single-lumen, camera-embedded ET in combination with a BB, might be the tool of choice for airway handling as it has shown speed and ease of use [7].

In the presented case, a situation that could easily have resulted in the surgery being postponed due to difficulties handling the airways and isolating the lung was resolved using this newly developed airway device including a tube with integrated video imaging device and light source at the distal end. In addition to providing information about any airway secretion or bleeding during surgery, this device can be a tool for continuous verification of BB placement and thereby eliminating the risk of dislodgement caused by movement of lung and mediastinal structures during surgery, abandoning the need to rely on auscultation alone. 


\section{References}

1. Popescu WM (2014) Advancements in Lung Isolation Techniques. Popescu WM. Advancements in Lung Isolation Techniques 37-45.

2. Shelley B, Macfie A, Kinsella J (2011) Anesthesia for thoracic surgery: a survey of UK practice. J Cardiothorac Vasc Anesth 25: 1014-7.

3. Narayanaswamy M, McRae K, Slinger P, Dugas G, Kanellakos GW, et al. (2009) Choosing a lung isolation device for thoracic surgery: a randomized trial of three bronchial blockers versus double-lumen tubes. Anesth Analg 108: 1097-101.

4. Neustein SM (2015) Pro: bronchial blockers should be used routinely for providing one-lung ventilation. J Cardiothorac Vasc Anesth 29: 234-6.

5. Brodsky JB (2015) Con: a bronchial blocker is not a substitute for a double-lumen endobronchial tube. J Cardiothorac Vasc Anesth 29: 237-9.

6. Smith GB, Hirsch NP, Ehrenwerth J (1986) Placement of double-lumen endobronchial tubes. Correlation between clinical impressions and bronchoscopic findings. Br J Anaesth 58: 1317-20.

7. Huitink JM, Koopman EM, Bouwman RA, Craenen A, Verwoert M, et al. (2013) Tracheal intubation with a camera embedded in the tube tip (Vivasight $\left({ }^{(\mathrm{N})}\right)$ ). Anaesthesia 68: 74-8.

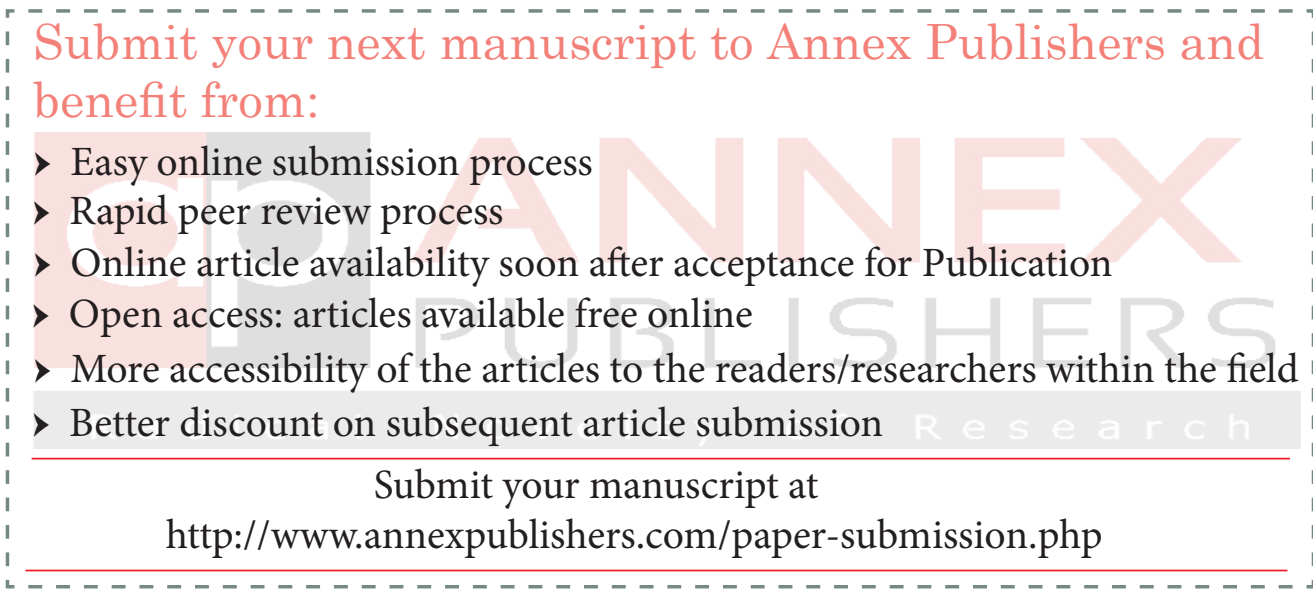

